\title{
LUTZOMYIA INFRASPINOSA (MANGABEIRA, 1941) (DIPTERA: PSYCHODIDAE) NUEVO REGISTRO PARA COLOMBIA, S.A.
}

\author{
ALBERTO MORALES*, CRISTINA FERRO*
}

El macho de Lutzomyia infraspinosa (Mangabeira, 1941) fue descrito por Octavio Mangabeira Filho (1) a partir de especímenes recolectados en Brasil así: 4 machos capturados en cueva de armadillo en Aurá, Belem, Pará, en agosto y septiembre de 1940; un macho recolectado en cueva de borugo, en noviembre de 1940 en la margen brasilera del río Mamoré, frontera Mato Grosso Bolivia, en la región del kilómetro 320 de la carretera Ferro Madeira-Mamoré; un macho capturado en Piratuba, municipio Abaeté, Pará, en octubre de 1938.

La hembra de Lutzomyia infraspinosa fue descrita por Floch \& Abonnenc (2) en 1943 basados en especímenes recolectados en la Guyana Francesa.

En 1987 Young \& Morales (3) comunicaron el hallazgo de una hembra de Lutzomyia capturada con trampa de luz (CDC) en la noche del 10-11 de febrero de 1986 a la orilla del río Iteviare, comisaría del Vichada y la cual se determínó como perteneciente a una de las dos especies, Lutzomyia begonae (Ortiz \& Torres, 1975) o Lutzomyia infraspinosa (Mangabeira, 1941) sin que se pudiera decidir cual de éstas era, puesto que las dos especies mencionadas son muy difíciles de separar específicamente cuando no se cuenta con la asociación de los respectivos machos.

En octubre 19 de 1989 en la finca La Primavera, vereda San Nicolás, municipio de Lebrija, Santander, Colombia, en captura nocturna de 7-8 p.m. en un bosque secundario y con cebo humano se recolectó un macho de Lutzomyia infraspinosa constituyéndose este registro en el primero, con seguridad, de esta especie en Colombia.

De acuerdo con Dedet (4), la Lutzomyia infraspinosa ha sido encontrada además de Brasil, en Guyana Francesa, Surinam, Bolivia, Perú y Venezuela.

La Lutzomyia infraespinosa pertenece al subgénero Evandromyia (Mangabeira) dentro del cual se conocen
7 especies: Lutzomyia bourrouli(Barretto \& Coutinho, 1941), Lutzomyia brachiphalla (Mangabeira, 1941), Lutzomyia cerqueirai (Causey \& Damasceno, 1945), Lutzomyia infraspinosa (Mangabeira, 1941), Lutzomyia inpai (Young \& Arias, 1977), Lutzomyia begonae (Ortiz \& Torres, 1975), Lutzomyia pinotti (Damasceno \& Arouck, 1956), Lutzomyia monstruosa (Floch \& Abonnenc, 1944) y Lutzomyia teratodes (Martins, Falcao \& Silva, 1964) (5).

En Colombia se han encontrado, hasta ahora, del subgénero Evandromyia las siguientes especies: $L u$. infraspinosa, Lu. monstruosa y $\mathrm{Lu}$. cerqueirai.

\section{AGRADECIMIENTOS}

A los señores Enrique Martínez y Amilcar González quienes recolectaron el material de $\mathrm{Lu}$. infraspinosa en Lebrija (Santander).

\section{BIBLIOGRAFIA}

1. Mangabeira O. 3a. Contribuicao ao estudo dos Flebotomus. Evandromyia n. subg. (Diptera: Psychodidae). Mem Instituto Oswaldo Cruz 1941 36: 215.

2. Floch $\mathrm{H}$, Abonnenc E. Phlebotomes de la Guyana Francaise, V. Institut Pasteur de la Guyane et du Territoire de L'Inini 1943; Publication No. 61: 1.

3. Young DG, Morales A. New species and records of Phlebotomine sand flies from Colombia (Diptera: Psychodidae). J Med Ent 1987; 24: 651 .

4. Dedet JP. Lista de especies de flebótomos presentes en los países andinos y las Guayanas. Red Latino-americana de entomólogos especializados en flebótomos. Boletín Informativo $1989 ; 4: 1$.

5. Young DG, Arias JR. Lutzomyia sand flies in the subgenus Evandromyia Mangabeira with descriptions of a new species from Brazil (Diptera: Psychodidae). Acta Amazónica 1977; 7: 59

*Grupo de Entomología. Instituto Nacional de Salud. A.A. 80080, Bogotá, Colombia, S.A. 